\title{
Perfil dos Demitidos durante a Crise de 2008 no Brasil
}

Fabio Jose Ferreira da Silva ${ }^{1}$

Resumo: O artigo analisa os efeitos da crise do subprime sobre o mercado de trabalho brasileiro. Através de um modelo de transição, que diferencia os demitidos da crise daqueles não demitidos, investiga características individuais e dos postos de trabalho relacionadas com a probabilidade de demissão. Encontramos que trabalhadores informais, subocupados, com menos tempo de serviço e menor rendimento tiveram maiores chance de demissão. Acerca das características dos indivíduos, as demissões foram maiores nos extremos da escala de idade - jovens e velhos - em comparação aos adultos; menores níveis de escolaridade aumentaram a probabilidade de demissão; mulheres foram mais atingidas que os homens. Os resultados sinalizam que crise acentuou as disparidades no mercado de trabalho.

Palavras-chave: Crise do subprime, Desemprego, Brasil

JEL: J21; J22; J23 


\title{
Profile of the Dismissed Workers During the 2008 Crisis in Brazil
}

\begin{abstract}
The paper analyzes the effects of the subprime crisis on the Brazilian labor market. We use a transition model, which distinguishes workers fired from jobs from those non-fired and we investigate individual attributes and characteristics of the jobs related to the likelihood of dismissal. About the characteristics of individuals, the layoffs were higher at the extremes of age range-young and old - in comparison to adults, lower levels of education increased the likelihood of dismissal, and women were more affected than men. The results indicate that the crisis has accentuated disparities in the labor market.
\end{abstract}

Keywords: Subprime Crisis. Unemployment. Brazil.

JEL: J21; J22; J23

\section{Introdução}

A crise financeira do subprime, originada no mercado hipotecário de alto risco norte americano, alastrou-se pelo mundo de maneira intensa e veloz, considerada por alguns como a pior crise financeira desde a Grande Depressão. Entre diversos artigos, foi objeto de análise por Stiglitz (2008, 2009), Krugman (2009), Eichengreen (2009), Reinhart e Rogoff (2009). Transmitida para a economia real, a crise implicou em demissões, ainda que a magnitude do choque tenha variado substancialmente entre os países. De maneira geral, os reflexos no mercado de trabalho foram mais duradouros nos países desenvolvidos em comparação aos países em desenvolvimento. De acordo com o International Labour Conference (2010), as heterogeneidades de seus efeitos decorreram de 3 razões principais:

(i) Situação do sistema financeiro doméstico antes da crise. Países onde o sistema se envolveu em operações de alto risco (como os derivativos complexos envolvendo títulos do mercado imobiliário) foram mais expostos à restrição ao crédito, comprometendo o fluxo de recursos ao setor privado;

(ii) Solidez fiscal. Países que entraram na crise com maior equilí- 
brio fiscal tiveram condições de implementar políticas contracíclicos e medidas de estímulo, em contraste a outros que tiveram menores condições de reação;

(iii) Intensidade do impacto externo. Sofreram mais as economias mais endividadas no mercado internacional, onde o setor exportador tem maior participação no PIB e no emprego, e/ou os investimentos externos são mais relevantes.

Analisando uma amostra de 84 países, o International Labour Conference (2010) enquadra o Brasil no grupo onde os efeitos sobre o desemprego foram considerados de leve a moderado.

Ainda são pouco numerosos os estudos que analisam os efeitos da crise no Brasil. Moretto e Proni (2011) relacionam os impactos menores da crise sobre o desemprego no Brasil, quando comparado a vários outros países, às políticas anticíclicas adotadas pelo governo, que privilegiaram a manutenção da demanda, garantindo o nível de atividade e do emprego. Entre elas, citam-se, na área monetária, a redução das taxas de juros, medidas para equilibrar a liquidez e o aumento da oferta de crédito dos bancos públicos. Na esfera fiscal houve desoneração tributária, ampliação dos investimentos em infraestrutura e das transferências aos programas sociais. Cacciamali e Tattei (2010) realçam o papel da redução da vulnerabilidade externa e a melhora dos fundamentos da economia nacional na década de 2000 que aumentaram a capacidade de resposta a choques adversos, em contraste com a década anterior.

No entanto, o fato do Brasil ter sido comparativamente menos afetado não deve ser interpretado como se tivesse sido imune. Silva e Fonseca-Neto (2011) mostra que os reflexos da crise não foram brandos, como se poderia supor pela elevação aparentemente modesta da taxa de desemprego (de 7,7\% em novembro de 2008 para 8,6\% em maio de 2009, com dados dessazonalizados). Comparando com outras duas fases de aumento do desemprego, a metodologia utilizada mostra que a redução da demanda por mão-de-obra foi a mais intensa verificada com os dados da Pesquisa Mensal do Emprego (PME/IBGE) desde março de 2002, quando se inicia sua metodologia atual. Em períodos anteriores, o aumento do desemprego resultou de um crescimento da demanda por mão-de-obra em ritmo inferior ao da oferta de mão de obra, diferentemente do ocorrido durante a crise do subprime, quando houve destruição líquida de postos de trabalho. Pochmann (2009) também encontra evidências de deterioração das condições do mercado de trabalho, como o aumento da informalidade e da rotatividade nos empregos assalariados formais, pela demissão de trabalhadores com maiores remunerações e contratação de trabalhadores com condições inferiores de salário.

O presente artigo utiliza os microdados da Pesquisa Mensal do Emprego (PME) do IBGE como fonte de dados. Uma característica interessante desta pesquisa é seu caráter longitudinal - isto é, o acompanhamento das mesmas 
pessoas ao longo do tempo - permitindo diferenciar os indivíduos que perderam os postos de trabalho no período da crise daqueles que permaneceram ocupados. A vantagem de se trabalhar nesse nível de desagregação é a garantia que as variações observadas não estão sendo provocadas pela troca de informantes. $\mathrm{O}$ artigo diferencia-se dos demais ao comparar esses dois grupos, em um modelo de transição, investigando quais características individuais dos ocupados (idade, sexo, escolaridade) e de seus postos de trabalho (formalidade, subocupação, tempo de serviço e rendimento do trabalho antes de ser demitido) estão relacionadas com o aumento da chance de demissão. A análise foi restrita ao setor industrial, haja vista que não foram identificados efeitos relevantes da crise sobre o setor de serviços. A agropecuária foi desconsiderada pela baixa presença nas regiões pesquisadas (inferior a 1\%).

O tema é de interesse dos gestores públicos, considerando-se que o melhor conhecimento da evolução do mercado de trabalho durante a crise fornece subsídios para que políticas públicas compensatórias sejam bem direcionadas, em resposta a eventuais choques futuros.

Nossos principais resultados obtidos foram: no que concerne às características individuais, as demissões foram maiores nos extremos da escala de idade - jovens e velhos - em relação aos adultos. Maiores níveis de escolaridade estiveram associados a menores probabilidades de demissão. Também encontramos que as mulheres foram mais atingidas que os homens. Acerca das características dos postos de trabalho, o grupo mais afetado foram os trabalhadores informais, subocupados, com menos tempo de serviço e menor rendimento. Percebe-se, portanto, que as demissões penalizar os trabalhadores em postos de trabalho menos protegidos e de menores custos de demissão, que podem ser substituídos com maior facilidade em caso de retomada da atividade econômica.

Além dessa introdução, o artigo está organizado em mais cinco seções. A seção seguinte apresenta a descrição dos efeitos da crise nos índices de ocupação setoriais. Em seguida, a segunda seção contém os modelos teórico e empírico. Os resultados das diferentes especificações estão na seção 3 e os testes de ajustamento do modelo constam na seção 4. Na seção 5 as principais conclusões são enunciadas.

\section{Análise descritiva}

Inicialmente, busca-se delimitar o período de incidência e as atividades atingidas pela crise. A definição do período de uma crise envolve certa sub- 
jetividade, sendo que se optou por um critério simples, baseado na evolução do total de ocupados medido pela PME. Os sessenta segmentos pesquisados foram agrupados em três setores: (i) indústria'; (ii) construção civil; (iii) serviços. As séries foram dessazonalizadas e atribuiu-se base 100 à média de 2005, conforme Gráfico 1.

\section{GRÁFICO 1 - OCUPAÇÃO DOS SETORES}

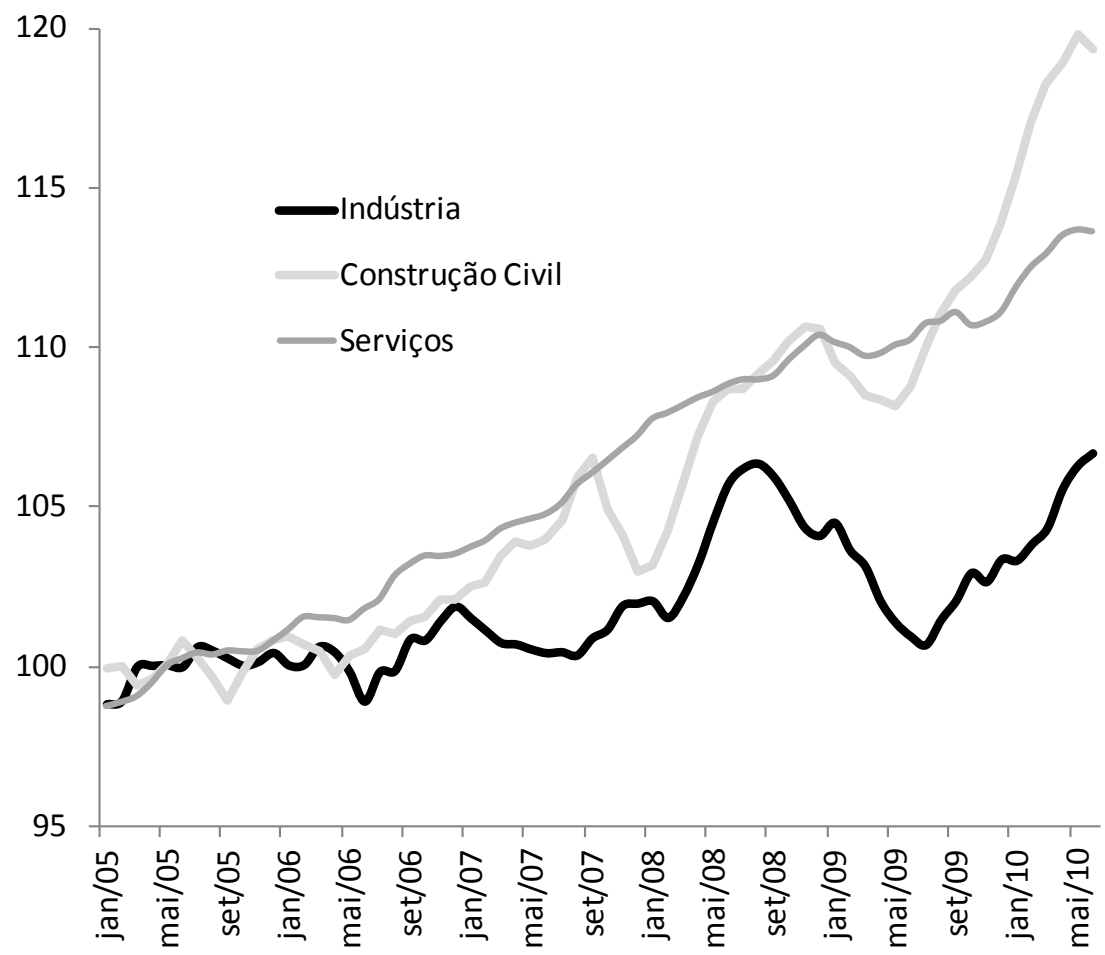

Fonte: PME/IBGE. Dados dessazonalizados. Média de $2005=100$.

Comparando a evolução da ocupação dos três setores nota-se que, para o setor industrial, no período de incidência do subprime a retração foi mais tempestiva (iniciou-se antes dos demais, em setembro de 2008), duradoura (10 meses) e intensa (até julho de 2009 o total de postos de trabalho recuou $4,9 \%)$. Na construção civil a ocupação começou a diminuir em novembro de 2008, atingindo nível 2,3\% menor em maio, mas em agosto a ocupação já havia recuperado o patamar pré-crise. Por outro lado, pode-se dizer que o 
setor de serviços passou ao largo da crise.

A evidência que a crise concentrou-se na indústria justifica a análise deste setor no presente artigo. O período de incidência foi definido da seguinte forma: inicia-se quando o nível de ocupação recua 3 meses consecutivos e termina quando este se eleva por 3 meses consecutivos, resultando nos meses de setembro (mês base) de 2008 a julho (inclusive) de $2009^{2}$.

Em valores absolutos, a perda de empregos da indústria totalizou 179,6 mil postos de trabalho, resultado da eliminação de 183,1 mil na indústria de transformação, parcialmente compensada pelos 1,9 mil empregos criados na indústria extrativa e 1,6 mil nos SIUP, como se observa no Anexo 1. Em termos percentuais, vários segmentos chegaram a perder mais de $10 \%$ do total de ocupados: metalurgia básica, 11,6\%, preparação de couro e calçados 13,7\%, produtos de metal, 16,7 e "fabricação de equipamentos de instrumentação médico-hospitalares, instrumento de precisão e ópticos, equipamentos para automação industrial”, $17,5 \%$.

\section{Metodologia}

\subsection{Modelo teórico}

A transição para o desemprego é analisada através de modelos de escolha binária, tendo em consideração que a variável explicativa é composta por apenas dois tipos de indivíduos: os que foram demitidos durante a crise e os que permaneceram ocupados. A princípio, pode-se partir de um modelo de probabilidade linear (MPL), especificado da seguinte forma:

$$
\mathrm{P}(\mathrm{y}=\mathbf{1} \mid \mathrm{x})=\beta_{\mathrm{o}}+\beta_{1} \mathrm{x}_{1}+\beta_{2} \mathrm{x}_{2}+\ldots \beta_{\mathrm{k}} \mathrm{x}_{\mathrm{k}}
$$

Em que $\beta_{\mathrm{j}}$ e $\mathrm{x}_{\mathrm{j}}$ são interpretados da maneira usual. O MPL, no entanto, não representa bem a probabilidade de resposta $\mathrm{P}(\mathrm{y}=\mathbf{1} \mid \mathrm{x})$, a menos que $\mathbf{x}$ esteja fortemente restrito. Para certos valores de $\beta_{\mathrm{j}}$, haveria valores de $\mathrm{x}_{1}, \ldots, \mathrm{x}_{\mathrm{l}}$, em que $\beta_{\mathrm{o}}+\mathrm{x} \beta$ estariam fora do intervalo compreendido por o e 1. Outra deficiência do MPL é que, ceteris paribus, o aumento de uma unidade em $\mathrm{x}_{\mathrm{j}}$

2 Reconhecemos que o critério é sujeito a críticas, por exemplo, é possível que os efeitos da crise tenham se manifestado antes do início das demissões, considerando-se que estes podem suceder medidas de contenção de oferta, como a concessão de férias coletivas e acordos entre patrões e empregados. Ao final do período, as contratações podem responder com defasagem à recuperação da atividade econômica. 
sempre aumenta $\mathbf{P}(\mathbf{y}=\mathbf{1} \mid \mathbf{x})$ no mesmo montante. Isso não pode ser sempre verdade porque aumentos (ou reduções) contínuos de $\mathrm{x}_{\mathrm{j}}$ eventualmente levariam $\mathrm{P}(\mathrm{y}=1 \mid \mathrm{x})$ a valores menores do que o ou acima de 1 . Ainda assim, o MPL geralmente é considerado uma aproximação da probabilidade de resposta para valores intermediários das variáveis independentes, vide Wooldridge (2002). Não sujeito às deficiências citadas, é comum a utilização de modelos logit, em que:

$$
\mathrm{p}(\mathrm{y}=\mathbf{1} \mid \mathrm{x})=\mathrm{G}(\mathrm{x} \beta) \equiv \mathrm{p}(\mathrm{x})
$$

Onde $\mathrm{G}$ representa a função de densidade acumulada que, no caso do logit, é dada pela função logística $(\Lambda)$.

$$
\mathrm{G}(\mathrm{z})=\Lambda(\mathrm{z}) \equiv \exp (\mathrm{z}) /[1+\exp (\mathrm{z})]
$$

No caso, os efeitos parciais dependem dos níveis das variáveis independentes. Para contínuo, temos que:

$$
\frac{\partial \mathrm{p}(\mathrm{x})}{\partial \mathrm{x}_{\mathrm{j}}}=\mathrm{g}(\mathrm{x} \beta) \text {, onde } \mathrm{g}(\mathrm{z}) \equiv \frac{\mathrm{dG}}{\mathrm{dz}}(\mathrm{z})
$$

O efeito de $\mathrm{x}_{\mathrm{i}}$ em $\mathrm{p}(\mathrm{x})$ depende de $\mathbf{x}$ através de $\mathrm{g}(\mathrm{x} \beta)$. Como a função de densidade acumulada $\mathrm{G}($.) é crescente, $\mathrm{g}(\mathrm{z})$ é positiva para todo z. Os parâmetros da regressão determinam o sentido do efeito parcial, mas isoladamente não medem a magnitude do efeito.

Para binário, o efeito parcial de quando varia de o a 1, mantendo todas as demais variáveis fixas é dado por:

$$
\mathrm{G}\left(\beta_{1}+\beta_{2}+\ldots+\beta_{\mathrm{k}-1} \mathrm{x}_{\mathrm{k}-1}+\beta_{\mathrm{k}}\right)-\mathrm{G}\left(\beta_{1}+\beta_{2}+\ldots+\beta_{\mathrm{k}-1} \mathrm{x}_{\mathrm{k}-1}\right)
$$


A base de dados utilizada é a PME, que é uma pesquisa mensal, iniciada em 1980, com periodicidade mensal. Desde sua implementação, a pesquisa passou por modificações, sendo que a metodologia atual inicia-se em março de 2002. São entrevistados cerca de 30 mil domicílios mensalmente e coletadas informações relativas ao mercado de trabalho de indivíduos a partir dos 10 anos de idade. As seguintes regiões metropolitanas são pesquisadas: Recife, Salvador, Belo Horizonte, Rio de Janeiro, São Paulo e Porto Alegre.

A PME é uma pesquisa em painel, em bases rotativas: os domicílios são entrevistados por 4 meses consecutivos, ficam 8 meses fora da entrevista e voltam a ser entrevistados por mais 4 meses. Ainda que a pesquisa não forneça um número para cada indivíduo (como seria o CPF ou RG por exemplo) é possível emparelhá-los de acordo com algumas características, seguindo procedimento descrito em Ribas e Soares (2008). O anexo 2 resume esta etapa de construção da base de dados.

Ao explorar o caráter longitudinal da pesquisa é possível diferenciar as pessoas que foram demitidas daquelas que permaneceram ocupadas durante o período de incidência da crise. Dessa forma, tem-se a garantia que as variações observadas não estão sendo provocadas pela troca de informantes.

\subsubsection{Especificações}

Nossa variável dependente assume dois valores, definidos da seguinte forma: 1 se o sujeito foi demitido e o se permaneceu ocupado. Os demitidos são aqueles que estiveram ocupados da indústria em $t$ e que perderam a ocupação em $t+1$, ou $t+2$ ou $t+3$. Os não demitidos são aqueles ocupados na indústria em $t$ e que permaneceram nessa condição por pelo menos 1 dos 3 meses subsequentes. Outros casos foram desconsiderados, incluindo as pessoas que foram entrevistadas uma única vez.

A análise empírica consiste na estimativa de influências de dois conjuntos de variáveis sobre a probabilidade de demissão dos ocupados da indústria. O primeiro grupo é composto por características individuais: idade, sexo (homem=1), cor (branco=1) e escolaridade. O segundo grupo contém atributos do posto de trabalho ${ }^{3}$ : se o vínculo empregatício é formal, se o trabalhador é subocupado, duração no emprego, desvio do rendimento em relação à média. Tais variáveis 
foram definidas da seguinte forma:

(i) Formalidade. Foram considerados empregados formais os empregadores e empregados com carteira assinada (dummy $=1$ ), enquanto os empregos informais são constituídos pelos empregados por conta própria, empregados sem carteira de trabalho assinada e trabalhadores não remunerados (dummy=0);

(ii) Subocupação. De acordo com a definição utilizada pelo IBGE, os trabalhadores subocupados são aqueles ocupados, que trabalharam menos de 40 horas semanais na semana de referência da pesquisa, estando disponíveis para trabalhar além do número de horas que efetivamente trabalharam, além de ter perspectiva de continuar nessa condição (dummy=1).

(iii) Duração no emprego. Foram mantidas as quatro faixas de duração no emprego pesquisadas na PME, correspondentes ao período de até 30 dias (grupo de referência), de 31 dias a menos de 1 ano, de 1 ano a menos de 2 anos e, por último, 2 anos ou mais.

(iv) Desvio do Rendimento em relação à média. Para um indivíduo $i$ no período $t$, o desvio do rendimento em relação à média é dado por onde $w$, representa o rendimento por hora trabalhada, medido em valores reais de setembro de 2009, inflacionados pelo INPC do IBGE.

Como variáveis de controle, são incluídas variáveis dummy de segmento, mês e região. As dummies de segmento se justificam porque os efeitos da crise foram heterogêneos entre os segmentos, com maior ênfase sobre aqueles compostos por empresas exportadores e/ou que possuem algum tipo de restrição ao crédito. Além disso, essa dummy suaviza efeitos de sazonalidade na contratação. As dummies de mês controlam por efeitos macroeconômicos que afetam indistintamente todos os segmentos. 
FIGURA 1 - ESPECIFICAÇÕES DOS MODELOS

\begin{tabular}{|c|c|c|c|c|}
\hline & Modelo 1 & Modelo 2 & Modelo 3 & Modelo 4 \\
\hline \multicolumn{5}{|l|}{$\begin{array}{l}\text { Características } \\
\text { individuais }\end{array}$} \\
\hline Idade & $\mathrm{x}$ & & $\mathrm{x}$ & $\mathrm{x}$ \\
\hline Sexo & $\mathrm{x}$ & & $\mathrm{x}$ & $\mathrm{x}$ \\
\hline Cor & $\mathrm{x}$ & & $\mathrm{x}$ & $\mathrm{x}$ \\
\hline Escolaridade & $\mathrm{x}$ & & $\mathrm{x}$ & $\mathrm{x}$ \\
\hline \multicolumn{5}{|l|}{$\begin{array}{l}\text { Características do } \\
\text { posto de trabalho }\end{array}$} \\
\hline Formal & & $\mathrm{x}$ & & \\
\hline Subocupado & & $\mathrm{x}$ & $\mathrm{x}$ & $\mathrm{x}$ \\
\hline $\begin{array}{l}\text { Duração no } \\
\text { Emprego }\end{array}$ & & $\mathrm{x}$ & $\mathrm{x}$ & $\mathrm{x}$ \\
\hline Rendimento & & $\mathrm{x}$ & $\mathrm{x}$ & $\mathrm{x}$ \\
\hline Dummies de setor & Sim & Sim & Não & Sim \\
\hline Dummies de mês & Sim & Sim & Não & Sim \\
\hline $\begin{array}{l}\text { Dummies de } \\
\text { região }\end{array}$ & Sim & Sim & Não & Sim \\
\hline
\end{tabular}

Foram utilizadas especificações alternativas de modelos. Ao estimar diferentes modelos, pretende-se verificar quais efeitos são robustos, ou seja, que se mantêm significativos mesmo com algumas alterações nas especificações. A princípio são estimados dois modelos - respectivamente modelo 1 e modelo 2 , conforme tabela abaixo - sendo que o modelo 1 contém apenas as características individuais e o modelo 2 apenas as variáveis dos postos de trabalho. Considerando que ambos os conjuntos de variáveis devem ser importantes, rodam-se os modelos 3 e 4, cuja diferença é que o modelo 3 não inclui as dummies de controle.

Além dos regressores supracitados, existem características não observadas que podem influenciar a probabilidade de demissão. Os gestores, ao selecionar quais trabalhadores demitir, podem ter levado em conta habilidades individuais. Assim, os mais habilidosos podem ser aqueles que permaneceram ocupados no período da crise. Esta característica pode estar correlacionada com as características dos postos de trabalho e/ou escolaridade e por isso 
deve-se ter cautela na interpretação dos resultados como efeitos causais.

\section{Resultados}

\subsection{Resultados preliminares}

A amostra é composta por 2.230 demitidos e 14.994 não demitidos que, expandidos pelos pesos amostrais, totalizam 896 mil e 7.185 mil. Antes de apresentar os efeitos marginais, comparam-se, na tabela 1, os dois grupos de interesse. A primeira coluna mostra, do total de demitidos, qual é o percentual que possui os atributos descritos nas linhas 4 . Por exemplo, dos demitidos, 47,6\% são homens, 55,4\% são brancos. A segunda coluna apresenta os valores para os não demitidos. A terceira coluna mede a razão entre a primeira e a segunda colunas, de modo que valores acima de 1 indicam que o atributo foi mais frequente entre os demitidos.

Destacam-se, entre os maiores valores da terceira coluna, o de 4,4 assinalado na duração da ocupação de até 30 dias e o de 4,0 no caso da subocupação. Isso significa que a fatia de empregados até 30 dias e de subocupados é maior entre os demitidos do que entre os não demitidos, sinalizando que essa característica pode ${ }^{5}$ estar relacionada ao aumento da probabilidade de demissão. No sentido oposto, evidencia-se que o rendimento médio dos demitidos é menor (valor de 0,5 observado na coluna 3), que há menos homens entre os demitidos e menos pessoas de 11 anos ou mais de estudo.

4 As exceções são as variáveis idade e rendimento, cujos valores são as médias, medidas, respectivamente, em anos e em moeda constante.

5 Apenas através da regressão - apresentada na seção seguinte - é que se isola o efeito do regressor sobre a probabilidade de demissão, na medida em que controla pelos demais regressores. 
TABELA 1 - COMPARATIVO DAS CARACTERÍSTICAS DE DEMITIDOS E NÃO DEMITIDOS

\begin{tabular}{|c|c|c|c|}
\hline & Demitidos (A) & Não demitidos (B) & Relação (A/B) \\
\hline \multicolumn{4}{|l|}{$\begin{array}{l}\text { Características } \\
\text { individuais }\end{array}$} \\
\hline $\begin{array}{l}\text { Gênero (\% de } \\
\text { homens) }\end{array}$ & 47,6 & 65,1 & 0,7 \\
\hline $\begin{array}{l}\text { Cor (\% de } \\
\text { brancos) }\end{array}$ & 55,4 & 59,3 & 0,9 \\
\hline $\begin{array}{l}\text { Idade média (em } \\
\text { número de anos) }\end{array}$ & 38,2 & 37,6 & 1,0 \\
\hline \multicolumn{4}{|l|}{$\begin{array}{l}\text { Escolaridade } \\
\text { média }\end{array}$} \\
\hline $\begin{array}{l}\text { Sem instrução a } \\
\text { menos de } 8 \text { anos } \\
\text { de estudo (\%) }\end{array}$ & 34,3 & 23,9 & 1,4 \\
\hline $\begin{array}{l}\text { De } 8 \text { a } 10 \text { anos de } \\
\text { estudo (\%) }\end{array}$ & 30,0 & 19,1 & 1,6 \\
\hline $\begin{array}{l}11 \text { ou mais anos } \\
\text { de estudo (\%) }\end{array}$ & 42,7 & 57,0 & 0,7 \\
\hline \multicolumn{4}{|l|}{$\begin{array}{l}\text { Características } \\
\text { do posto de } \\
\text { trabalho }\end{array}$} \\
\hline $\begin{array}{l}\text { Informalidade } \\
\text { (\%) }\end{array}$ & 55,2 & 25,0 & 2,2 \\
\hline Subocupação (\%) & 4,4 & 1,1 & 4,0 \\
\hline $\begin{array}{l}\text { Rendimento } \\
\text { médio }(\mathrm{R} \$)^{*}\end{array}$ & 772,7 & $1.503,7$ & 0,5 \\
\hline \multicolumn{4}{|l|}{$\begin{array}{l}\text { Duração na } \\
\text { ocupação }\end{array}$} \\
\hline Até 30 dias (\%) & 5,7 & 1,3 & 4,4 \\
\hline $\begin{array}{l}\text { De } 31 \text { dias a } \\
\text { menos de } 1 \text { ano } \\
\text { (\%) }\end{array}$ & 29,0 & 16,1 & 1,8 \\
\hline $\begin{array}{l}\text { De } 1 \text { ano a menos } \\
\text { de } 2 \text { anos (\%) }\end{array}$ & 11,7 & 11,4 & 1,0 \\
\hline $\begin{array}{l}2 \text { anos ou } \\
\text { mais (\%) }\end{array}$ & 53,5 & 71,0 & 0,8 \\
\hline
\end{tabular}

Fonte: Elaboração própria com dados da PME. * Valores constantes de setembro de 2009, inflacionados pelo INPC. 


\subsection{Resultados}

TABELA 2 - MODELOS LOGIT - EFEITOS MARGINAIS (VALORES MULTIPLICADOS POR 100)
(1)
(2)
(3)
(4)

Características

individuais

\begin{tabular}{|c|c|c|c|}
\hline Idade & $-1,29^{* *}$ & $-0,81^{* *}$ & $--0,75^{* *}$ \\
\hline & {$[0,00]$} & {$[0,00]$} & {$[0,00]$} \\
\hline \multirow[t]{2}{*}{$\begin{array}{l}\text { Idade ao } \\
\text { quadrado }\end{array}$} & $+0,02^{* *}$ & $+0,01^{* *}$ & $+0,00^{* *}$ \\
\hline & {$[0,00]$} & {$[0,00]$} & {$[0,00]$} \\
\hline \multirow[t]{2}{*}{$\begin{array}{c}\text { Sexo } \\
\text { Masculino } \\
\end{array}$} & $-5,66^{* *}$ & $-2,65^{* *}$ & $-3,05^{* *}$ \\
\hline & {$[0,00]$} & {$[0,00]$} & {$[0,00]$} \\
\hline \multirow[t]{2}{*}{ Cor Branca } & $0,11^{* *}$ & $-0,10^{* *}$ & $-0,37^{* *}$ \\
\hline & {$[0,00]$} & {$[0,02]$} & {$[0,00]$} \\
\hline \multirow{2}{*}{$\begin{array}{l}\text { Escolaridade } \\
(8-10)\end{array}$} & $-1,30^{* *}$ & $-0,71^{* *}$ & $-0,53^{* *}$ \\
\hline & {$[0,00]$} & {$[0,00]$} & {$[0,00]$} \\
\hline \multirow[t]{2}{*}{$\begin{array}{l}\text { Escolaridade } \\
\text { (11 ou mais) }\end{array}$} & $-3,70^{* *}$ & $-1,47^{* *}$ & $-1,04^{* *}$ \\
\hline & {$[0,00]$} & {$[0,00]$} & {$[0,00]$} \\
\hline
\end{tabular}

Características

do posto de

trabalho

\begin{tabular}{|c|c|c|c|}
\hline Informal & $8,19^{* *}$ & $6,65^{* *}$ & $6,19^{* *}$ \\
\hline & {$[0,00]$} & {$[0,00]$} & {$[0,00]$} \\
\hline \multirow[t]{2}{*}{ Subocupado } & $5,51^{* *}$ & $9,16^{* *}$ & $5,14^{* *}$ \\
\hline & {$[0,00]$} & {$[0,00]$} & {$[0,00]$} \\
\hline \multicolumn{4}{|l|}{$\begin{array}{l}\text { Duração do } \\
\text { Emprego }\end{array}$} \\
\hline $\begin{array}{l}\text { (31 dias a } \\
\text { menos de } 1 \\
\text { ano) }\end{array}$ & $-3,43^{* *}$ & $-4,30^{* *}$ & $-3,37^{* *}$ \\
\hline (Continua) & & & \\
\hline
\end{tabular}




\begin{tabular}{|c|c|c|c|c|}
\hline \multicolumn{5}{|l|}{ (Continuação) } \\
\hline & (1) & (2) & (3) & (4) \\
\hline & & {$[0,00]$} & {$[0,00]$} & {$[0,00]$} \\
\hline \multicolumn{5}{|l|}{$\begin{array}{l}\text { Duração do } \\
\text { Emprego }\end{array}$} \\
\hline \multirow[t]{2}{*}{$\begin{array}{l}\text { (de } 1 \text { ano a } \\
2 \text { anos) }\end{array}$} & & $-4,89^{* *}$ & $-5,59^{* *}$ & $-4,74^{* *}$ \\
\hline & & {$[0,00]$} & {$[0,00]$} & {$[0,00]$} \\
\hline \multicolumn{5}{|l|}{$\begin{array}{l}\text { Duração do } \\
\text { Emprego }\end{array}$} \\
\hline \multirow[t]{2}{*}{$\begin{array}{l}(2 \text { anos ou } \\
\text { mais })\end{array}$} & & $-11,17^{* *}$ & $-13,76^{* *}$ & $-11,19^{* *}$ \\
\hline & & {$[0,00]$} & {$[0,00]$} & {$[0,00]$} \\
\hline \multirow[t]{2}{*}{$\begin{array}{c}\text { Ln } \\
\text { (Desvio do } \\
\text { Rendimento) } \\
\end{array}$} & & $-1,37^{* *}$ & $-1,21^{* *}$ & $-1,00^{* *}$ \\
\hline & & {$[0,00]$} & {$[0,00]$} & {$[0,00]$} \\
\hline \multirow[t]{2}{*}{$\begin{array}{l}\quad \text { Ln } \\
\text { (Desvio do } \\
\text { Rendimento) } \\
\text { ao quadrado }\end{array}$} & & $1,27^{* *}$ & $1,22^{* *}$ & $1, \mathrm{OO}^{* *}$ \\
\hline & & {$[0,00]$} & {$[0,00]$} & {$[0,00]$} \\
\hline $\begin{array}{l}\text { Dummies } \\
\text { de setor }\end{array}$ & SIM & SIM & NÃO & SIM \\
\hline $\begin{array}{l}\text { Dummies } \\
\text { de mês }\end{array}$ & SIM & SIM & NÃO & SIM \\
\hline $\begin{array}{l}\text { Dummies } \\
\text { de região }\end{array}$ & SIM & SIM & NÃO & SIM \\
\hline \multicolumn{5}{|l|}{ Observações } \\
\hline R-quadrado & 12,8 & 15,7 & 11,0 & 17,4 \\
\hline
\end{tabular}

Fonte: Microdados da PME de setembro de 2008 a julho de 2009.

Notas: A variável dependente vale 1 se o indivíduo foi demitido no período da crise e o se permaneceu empregado por pelo menos dois meses.

Os erros-padrão robustos a correlação dos resíduos são mostrados entre colchetes (multiplicados por 100). Níveis de significância $\left({ }^{*}\right) 5 \%$ e $(* *) 1 \%$.

As categorias omitidas, no caso de variáveis binárias é o complemento. Por exemplo, em sexo aparece masculino, portanto foi omitido o feminino. Demais variáveis omitidas: (i) escolaridade: sem instrução a menos de 8 anos de estudo; (ii) duração do emprego: até 30 dias. 
Os resultados obtidos foram:

Idade. Os modelos 1, 3 e 4 encontram uma relação não linear para o efeito da idade sobre a probabilidade de ser demitido. Utilizando o modelo 4, simulamos, no gráfico 2, a probabilidade de demissão para diferentes valores da idade de um indivíduo com as características médias da população (agente representativo). Percebe-se que os mais jovens e os mais velhos foram os mais atingidos e que a idade menos atingida foi de aproximadamente 40 anos.

Sexo. A probabilidade de demissão das mulheres foi superior a dos homens em 3,05 pontos percentuais (p.p.), conforme se verifica no modelo 4.

\section{GRÁFICO 2 - PROBABILIDADE DE DEMISSÃO E IDADE}

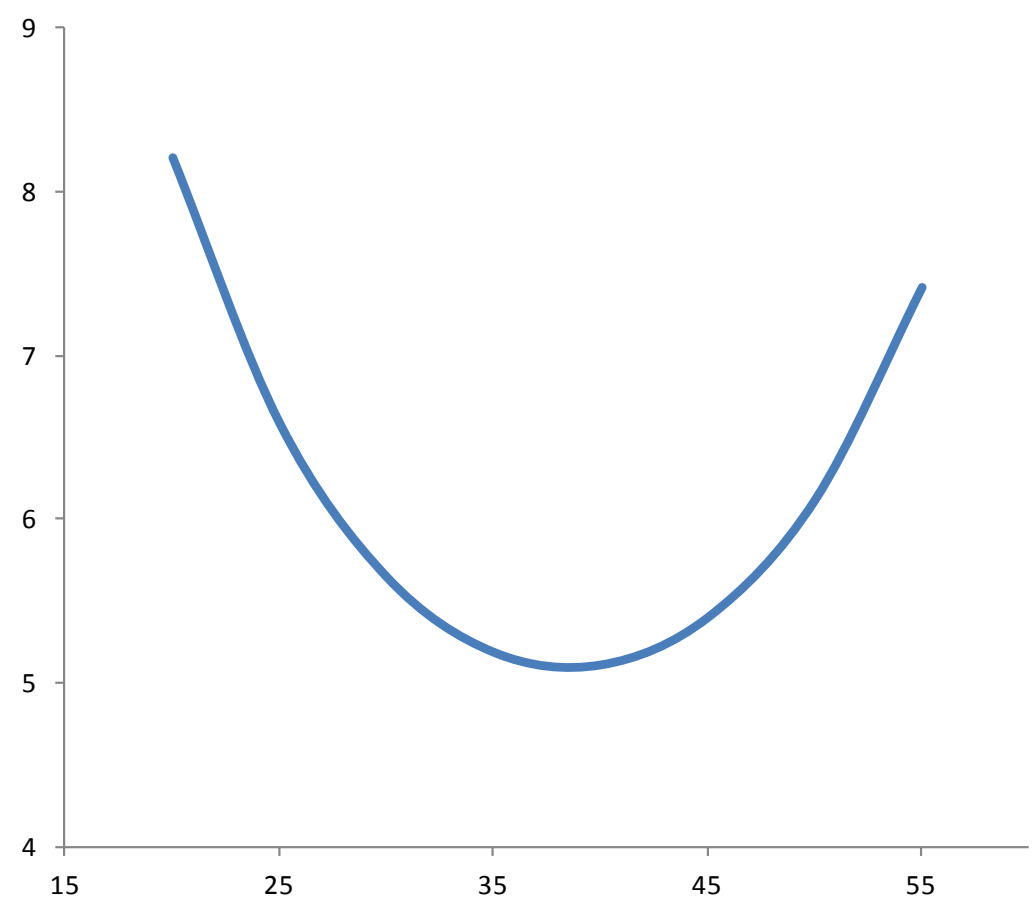

Fonte: Elaboração própria. Probabilidade multiplicada por 100.

Cor. Os brancos foram menos afetados em relação aos não brancos. O resultado deve ser analisado com ressalva pela pequena magnitude (o,37 p.p.) e pelo fato de seu sinal, no modelo 4, ter sido contrário ao encontrado no modelos 1.

Escolaridade. As pessoas com 8-10 anos de estudo, equivalente ao nível fun- 
damental completo ou o nível médio incompleto tiveram redução de o,53 p.p. da probabilidade de demissão em comparação ao grupo dos que não estudaram ou estudaram menos de 8 anos. Os indivíduos com 11 anos ou mais de estudo - equivalente ao nível superior incompleto ou completo - tiveram redução de 1,04 p.p. no mesmo tipo de comparação. Considerando-se a correlação positiva entre escolaridade e rendimento, a omissão do rendimento, no modelo 1, superestima o efeito da escolaridade. A "proteção" ao desemprego exercida pela conclusão do nível fundamental totalizou 1,30 p.p e a do nível médio 3,70 p.p..

Formalidade. Os empregados informais tiveram 6,19 p.p. mais chance de ser demitidos do que aqueles com vínculos formais. A ausência de custo de demissão dos trabalhadores informais deve ter influenciado o resultado.

Subocupação. No caso dos trabalhadores subocupados, a probabilidade de demissão foi 5,14 p.p. maior. Dado que os trabalhadores subocupados são, via de regra, informais, o aumento assinalado na probabilidade de demissão, controlando pela informalidade, é uma evidencia de que esse grupo é ainda mais vulnerável do que a média dos informais.

Duração no emprego. O tempo de emprego é um indicativo da experiência na função, de modo que indivíduos com 1 a 2 anos de emprego tiveram 4,74 p.p. menos probabilidade de demissão do que aqueles empregados até 30 dias. O menor custo de demissão de recém-admitidos também concorre para explicar o efeito. Os empregados a mais de 2 anos tiveram 11,19 p.p. menos de chance de ser demitidos.

Rendimento. Encontramos relação não linear entre o desvio do rendimento em relação à média e a probabilidade de demissão, vide Gráfico 3. Ocupados com rendimentos mais baixos tiveram maior chance de perder o emprego, tendência essa que fica menos intensa para níveis mais altos de salário. A título de exemplo, a probabilidade de demissão de um indivíduo que ganhava o salário mediano foi de $5,2 \%$, valor que se reduz a $4,5 \%$ para aqueles que ganhavam dois salários medianos.

Os modelos da Tabela 1 foram reestimados através de modelos MPL (vide apêndice 3) cujos resultados mostram-se similares aos descritos anteriormente. Nos quatro modelos reestimados todas as variáveis relativas ao posto de trabalho permaneceram significantes. As diferenças foram obtidas das variáveis individuais relativas a cor e escolaridade (controlando pelo rendimento), que se tornaram insignificantes aos padrões usuais. 


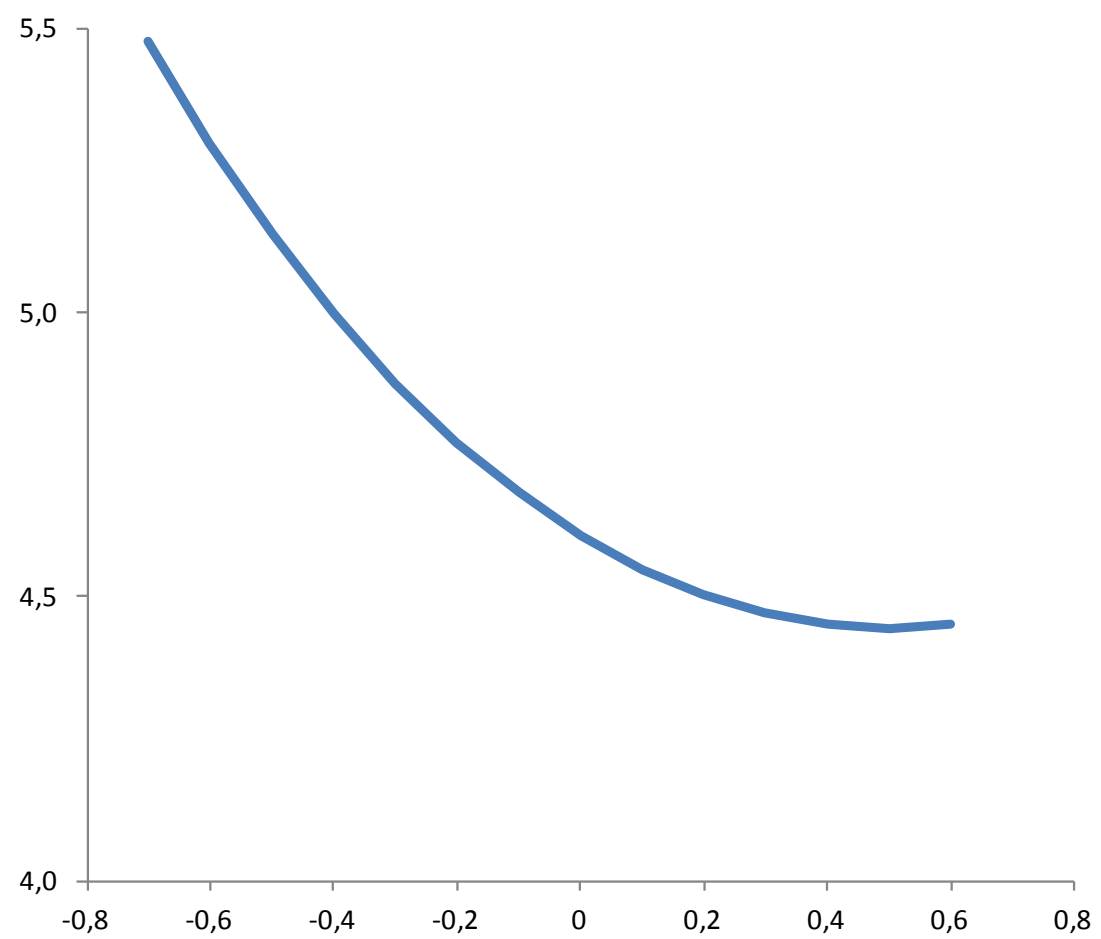

Fonte: Elaboração própria. Probabilidade multiplicada por 100.

\section{Testes e ajustamento do modelo}

O modelo foi estimado através de Máxima Verossimilhança e o algoritmo de maximização precisou de 4 interações para convergir. $O$ valor da razão de verossimilhança, que testa a hipótese nula de que todos os coeficientes das variáveis explicativas são iguais a zero, é realizado por meio de um teste Qui-Quadrado cujo p-valor é igual a o,0oo, levando à rejeição da hipótese nula.

A significância estatística de que cada coeficiente é igual a zero é realizada através de testes de Wald. Da mesma forma que o teste $t$ aplicado aos modelos 
lineares, a hipótese nula do teste de Wald é se o parâmetro estimado é igual a zero. A estatística Wald tem distribuição Qui-Quadrado, sendo calculada pela razão entre o coeficiente e o seu erro padrão. A tabela 1 apresenta as estimativas dos parâmetros das variáveis do modelo, os erros padrão e indicativos do p-valor.

Acerca das medidas de ajuste do modelo, inicialmente apresentam-se os valores de pseudo-R2 dos modelos 1 a 4, respectivamente: 12,8\%, 15,7\%, 11,0\% e $17,4 \%$. Outra medida, mais interessante quando se trabalha com modelos de escolha binária, é o percentual previsto corretamente pelo modelo, que fornece o percentual corretamente predito de 1's, quando a probabilidade é maior do que um determinado valor crítico; e de zeros, quando a probabilidade é menor que este valor. A escolha do valor crítico é feita em função de dois conceitos: (i) sensitividade, que é a proporção de acerto na previsão da ocorrência de um evento nos casos em que ele de fato ocorreu e (ii) especificidade, que mede a proporção de acerto na previsão da não ocorrência de um evento nos casos em que ele de fato não ocorreu.

Os conceitos variam em sentidos opostos em função do valor crítico, conforme se verifica no Gráfico 4, aplicado ao modelo 4. Para valores críticos próximos a zero, o modelo estimaria uma proporção muito elevada de demitidos. Por um lado, isso faria com a sensitividade fosse alta, porém a especificidade seria muito baixa, comprometendo a capacidade do modelo de diferenciar os demitidos dos nãodemitidos. A princípio, um valor de referência que pode ser utilizado como valor crítico é a frequência relativa empírica do total de demitidos de nosso banco de dados, que é de 10\%. O Gráfico 4 corrobora o valor crítico de $10 \%$ como equilibrado, na medida em que as curvas de sensibilidade e especificidade se cruzam nas proximidades deste ponto.

Para esse valor crítico, o modelo previu corretamente 70,7\% das observações. A proporção de valores corretamente previstos para os demitidos foi de $71,8 \%$ e a proporção de valores corretamente previstos para os não demitidos totalizou $70,5 \%$. 


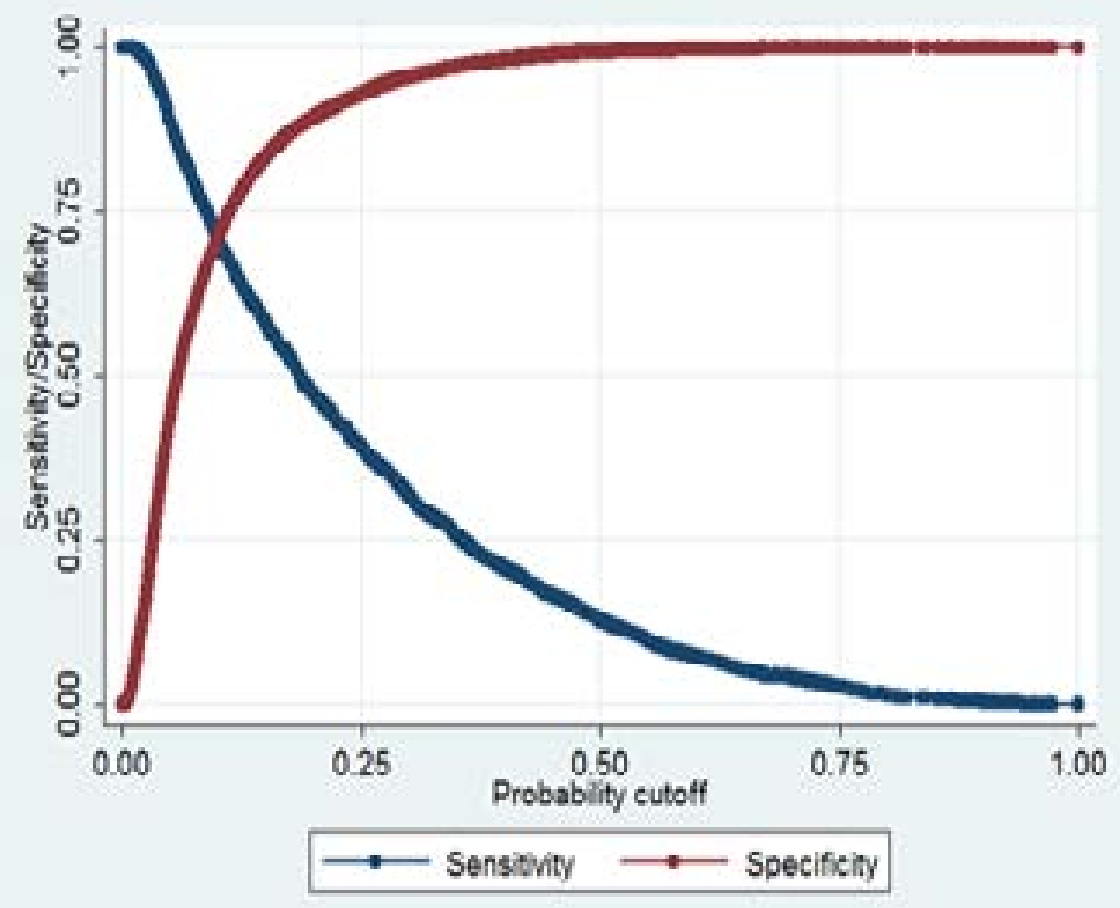

Fonte: Elaboração própria.

Um procedimento complementar utilizado para avaliar a performance do modelo é realizado através da construção de uma Curva ROC (Receiver Operating Characteristic). Para isso, a sensitividade e a especificidade são calculadas para todas as observações da amostra, considerando diferentes pontos de corte do modelo. A curva é obtida registrando em um gráfico "sensitividade" x " 1 - especificidade" para diversos pontos de corte, sendo comparada com a reta de $45^{\circ}$, que representa um modelo que não tem capacidade explicativa (a chance de uma observação qualquer pertencer a um grupo é de 50\%). A área sob a curva mede a capacidade de discriminação do modelo, ou seja, quanto mais próxima a curva estiver do ponto $(1,0)$, mais ajustado é o modelo. Hosmer e Lemeshow (2000) apresentam uma regra geral para avaliação do resultado da área sob a Curva ROC: a) área no intervalo entre 0,7 e 0,8: discriminação aceitável; b) área no intervalo entre o,8 e 0,9: excelente discriminação; c) área acima de 0,9: excepcional discriminação. A Curva ROC do modelo, representada no Gráfico 5 , revela que a área sob a curva é de 0,79 , com um intervalo de confiança de 0,78 e o,80, isto é, indicam um poder de discriminação do modelo aceitável ou excelente segundo a escala proposta, tais valores. 


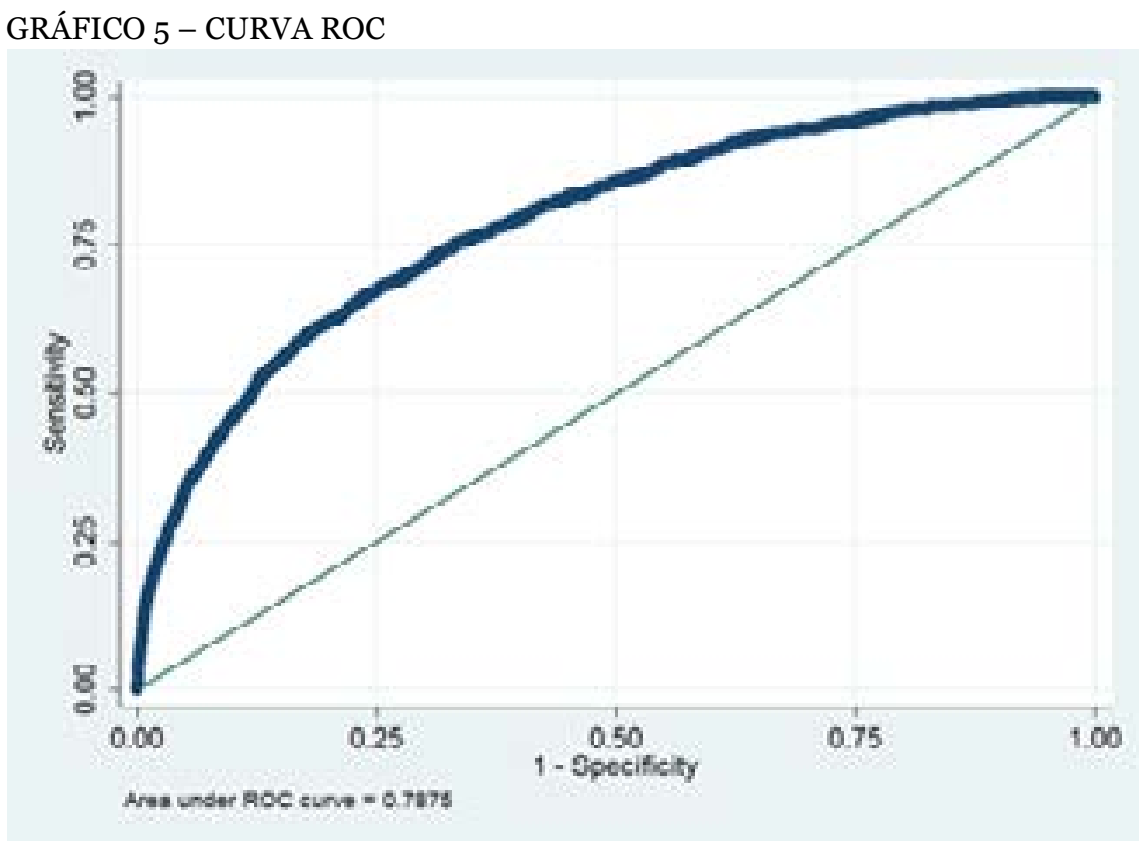

Fonte: Elaboração própria.

Contudo, conforme assinala Wooldridge (2002), em modelos de escolha binária, medidas de ajustamento de modelos não são tão importantes como a significância econômica e estatística das variáveis explicativas.

\section{Considerações Finais}

A crise do subprime impôs um choque de demanda profundo à indústria brasileira. Além da redução da demanda por produtos exportados, a restrição das fontes de financiamento e a deterioração das perspectivas de consumidores levaram a necessidade de reduzir a produção, implicando em demissões em larga escala. Setores como os de metalurgia básica e produtos de metal chegaram a perder mais de $10 \%$ da força de trabalho em apenas 10 meses.

Nesse ambiente, o presente artigo investiga o perfil dos demitidos na crise, buscando identificar quais características pessoais e dos postos de trabalho estiveram associados a maior probabilidade de demissão. Encontramos que, em relação às características dos indivíduos, as demissões foram maiores nos extremos da escala de idade - jovens e velhos - em relação aos adultos; menores níveis de escolaridade aumentaram a probabilidade de demissão; mulheres 
foram mais atingidas que os homens. Acerca das características dos postos de trabalho, o trabalho informal, subocupado, com menos tempo de serviço e menor rendimento aumentaram a chance de demissão.

Percebe-se, portanto, que as demissões penalizaram os trabalhadores em postos de trabalho menos protegidos e de menores custos de demissão, provavelmente porque podem ser substituídos com maior facilidade em caso de retomada da atividade econômica. Dessa forma, a crise acentuou as disparidades no mercado de trabalho.

\section{Referências Bibliográficas}

Cacciamali, M. C.; Tatei, F. Crise Econômica Mundial: mudança nas Características do Desemprego no Mercado de Trabalho? In: Moretto, A.; Krein, J. D.; Pochmann, M.; Macambira, J. (Orgs.) Economia, desenvolvimento regional e Mercado de trabalho no Brasil. Fortaleza-CE: IDT; BNB; Cesit, 2010. p. 53-78.

Eichengreen, B. The Financial Crises and Global Policy Reforms. Paper for the Federal Reserve Bank of San Francisco conference on Asia and the Financial Crisis. Santa Barbara, California. 2009.

Hosmer, David W.; Lemeshow, Stanley. Applied logistic regression. 2nd ed. New York: John Wiley \& Sons. 2000.

International Labour Conference. Recovery and Growth with Decent Work. $99^{\text {th }}$ Session. Report of the Director-General. Geneva. 2010.

Krugman, P. A crise de 2008 e a Economia da Depressão. Editora Campos. Rio de Janeiro. 2009.

Moretto, A.; Proni, M. W. O Desemprego no Brasil: Análise da Trajetória Recente. Economia e Desenvolvimento, Recife (PE), v. 10, n. 1, p.7-35. 2011.

Pochmann, M. O trabalho na crise econômica no Brasil: primeiros sinais. Estudos Avançados, vol.23, $\mathrm{n}^{0}$ 66. p.41-52. 2009.

Ribas, R; Soares, S. Sobre o painel da pesquisa Mensal do Emprego (PME) do IBGE. Ipea, 2008. (Texto para discussão, n. 1.348).

Reinhart C. M. \& Rogoff, K. S. This Time Is Different: Eight Centuries of Financial Folly. 2009.

Silva, F. J. F.; Fonseca-Neto, F. A. "A Crise do Subprime Alcança o Brasil: Canais de Transmissão e Efeitos Sobre o Desemprego". Anais do XII Encontro da Associação Brasileira de Estudos do Trabalho (ABET). 2011.

Stiglitz, J. E. "The Financial Crisis of 2007/2008 and its Macroeconomic Consequences", Meeting of the Initiative for Policy Dialogue Task Force meeting on 
SILVA, F. J. F. da. Perfil dos Demitidos da Indústria Metropolitana Durante a Crise de 2008 no Brasil Financial Markets Reform, junho. 2008.

Stiglitz, J. E. "The Current Economic Crisis and Lessons for Economic Theory", Eastern Economic Journal, 35, 281-295. 2009.

Wooldridge, J. M. "Econometric Analysis of Cross Section and Panel Data". The MIT Press. 2002. 